\title{
Les troubles cognitifs et comportementaux des traumatisés crâniens. Sans oublier le rachis et les AVC !
}

\author{
P. Pradat-Diehl \\ (C) Springer-Verlag 2009
}

Même si la politique de sécurité routière a certainement réduit le nombre des traumatismes crâniens (TC) par accident de la voie publique, les séquelles neurologiques des TC restent un problème grave de santé publique.

Les troubles cognitifs et comportementaux sont les séquelles les plus invalidantes des TC, et Philippe Azouvi actualise nos connaissances sur ces séquelles complexes. Ils sont en majorité à rapporter à un dysfonctionnement frontal et se manifestent par des troubles des fonctions exécutives, de l'attention et de la mémoire de travail, de la mémoire épisodique. Catherine Thomas-Antérion étudie, dans ce dossier, les capacités de patients à apprendre et à utiliser les nouveaux mots.

Les conséquences comportementales sont parfois explosives, confrontant les patients et leur entourage à un comportement d'agitation et d'agressivité. Faut-il avoir recours à des traitements médicamenteux ? Cette question polémique est traitée par Isabelle Richard et son équipe.

Les troubles du comportement ne sont pas uniquement neurologiques, et les troubles psychiatriques sont extrêmement fréquents dans cette population comme l'expose Hélène Oppenheim. Cette approche doit être connue, d'autant qu'elle nous est souvent moins familière que l'approche neurologique. Les concepts d'identité enrichissent notre approche des patients. Des suivis psychothérapeutiques sont proposés. Une consultation de recours devrait être proposée en Île-de-France pour répondre aux spécificités de l'approche psychiatrique et cognitive des troubles psychiques après TC.

Ces dernières années, l'intérêt s'est porté sur les TC de l'enfant. Mathilde Chevignard présente, ici, une mise au point extrêmement complète des séquelles gravissimes des TC survenus dans l'enfance. Plus jamais, après avoir lu cet article, vous ne pourrez rester sur l'impression lénifiante que la plasticité cérébrale protège l'enfant des séquelles ! Vous saurez que leurs séquelles sont au contraire encore plus graves que pour l'adulte, puisque le traumatisme retentit sur les capacités de maturation cérébrale et sur les apprentissages.

La description des limitations d'activité reliées à des troubles des fonctions organiques cognitives est au cœur de l'accompagnement des patients dans les programmes de réadaptation ou sert de base à la mise en place de moyens de compensation. Le rôle des ergothérapeutes est primordial pour évaluer les limitations d'activité. Rappelons à cette occasion que les ergothérapeutes ne sont pas uniquement des spécialistes de l'appareillage, leur place est essentielle dans l'évaluation et la rééducation des troubles des fonctions cognitives, dont les conséquences sont tout aussi concrètes que dans les limitations d'activité d'origine motrice. Ces situations écologiques peuvent démasquer ce fameux handicap invisible.

Ce dossier n'est qu'un aperçu de l'approche complexe des traumatisés crâniens et de leur entourage !

Du TC au rachis, il n'y a qu'une vertèbre, et je profite de cet éditoral pour saluer l'organisation de la Journée ANMSR 2009 sur le « Rachis cervical vieillissant », et remercier les organisateurs Jean-Claude Goussard et notre président Samy Bendaya. La Journée de mai 2010 reviendra vers le cerveau : nous y traiterons, avec Marc Genty, Béatrice Davenne, Frédérique Le Breton les actualités en rééducation de l'AVC. Nous vous attendons nombreux.

P. Pradat-Diehl

Hôpital de La Salpêtrière, 47, boulevard de l'Hôpital,

F-75013 Paris, France 